\title{
Editorial
}

\section{Some Issues in Liquid Metals Research}

\section{Maria José Caturla ${ }^{1,2, *}$, Jian-Zhong Jiang ${ }^{3, *}$, Enrique Louis ${ }^{1,2, *}$ and José Miguel Molina ${ }^{1,2,4, *}$}

1 Instituto Universitario de Materiales de Alicante (IUMA) and Unidad Asociada CSIC-UA, Universidad de Alicante, Ap. 99, E-03080 Alicante, Spain

2 Departamento de Física Aplicada, Universidad de Alicante, Ap. 99, E-03080 Alicante, Spain

3 International Center for New-Structured Materials (ICNSM), Department of Materials Science and Engineering, Zhejiang University, Hangzhou 310027, China

4 Departamento de Química Inorgánica, Universidad de Alicante, Ap. 99, E-03080 Alicante, Spain

* Authors to whom correspondence should be addressed; E-Mails: mj.caturla@ua.es (M.J.C.); jiangjz@zju.edu.cn (J.-Z.J.); enrique.louis@ua.es (E.L.); jmmj@ua.es (J.M.M.).

Received: 10 November 2015 / Accepted: 10 November 2015 / Published: 13 November 2015

The ten articles [1-10] included in this Special Issue on "Liquid Metals" do not intend to comprehensively cover this extensive field, but, rather, to highlight recent discoveries that have greatly broadened the scope of technological applications of these materials. Improvements in understanding the physics of liquid metals are, to a large extent, due to the powerful theoretical tools in the hands of scientists, either semi-empirical [1,5,6] or ab initio (molecular dynamics, see [7]). Surface tension and wetting at metal/ceramic interfaces is an everlasting field of fundamental research with important technological implications. The review of [2] is broad enough, as the work carried out at Grenoble covers almost all interesting matters in the field. Some issues of interest in geophysics and astrophysics are discussed in [3]. The recently discovered liquid-liquid transition in several metals is dealt with in [4]. The fifth contribution [5] discusses the role of icosahedral superclusters in crystallization. In [6], thermodynamic calculations are carried out to identify the regions of the ternary phase diagram of $\mathrm{Al}-\mathrm{Cu}-\mathrm{Y}$, where the formation of amorphous alloys is most probable. Experimental data and ab initio calculations are presented in [7] to show that an optimal microstructure is obtained if $\mathrm{Mg}$ is added to the Al-Si melt before than the modifier AlP alloy. Shock-induced melting of metals by means of laser driven compression is discussed in [8]. With respect to recent discoveries, one of the most outstanding developments is that of gallium alloys that are liquid at room temperature [9], and that, due to the oxide layer that readily cover their surface, maintain some "stiffness". This has opened the possibility of 3D printing with liquid metals. The last article in this Special Issue [10] describes nano-liquid metals, a suspension of liquid metal and its alloy containing nanometer-sized particles. A room-temperature nano-liquid metal and its alloys were first introduced in the area of cooling high heat flux devices, which now is a commercial reality. However, their applications are not only in chip 
cooling, and can also be extended to waste heat recovery, kinetic energy harvesting, thermal interface material, etc. This is mainly due to properties such as low melting point, high thermal and electrical conductivity, as well as other additional physical or chemical properties. These articles are summarized in more detail hereafter.

After having pointed out that, contrary to the gaseous and solid states, developing simple models of liquid metals that can lead to semi- or even quantitative predictions, involve serious difficulties not yet satisfactorily overcome, Wang [1] starts describing his proposal. The author shows that the energy sate of liquids may be approximated reasonably well by the energy and volume of a vacancy. Specifically, the idea is to approximate all forces exerted on each atom of the liquid using the energy needed to produce a vacancy. The author illustrates the adequacy of his simple model, calculating a variety of magnitudes and comparing his results with available experimental data. In particular, he presents data for the latent heat of melting for $\mathrm{Si}, \mathrm{Ge}, \mathrm{Bi}, \mathrm{Sb}, \mathrm{In}, \mathrm{Ar}, \mathrm{Kr}$, and $\mathrm{Xe}$, the volume changes on melting for $\mathrm{Si}, \mathrm{Ge}, \mathrm{Bi}, \mathrm{Sb}, \mathrm{Pu}, \mathrm{Ce}, \mathrm{La}, \mathrm{Tl}, \mathrm{Eu}, \mathrm{Yb}$, and Er, vacancy formation energy for around thirty metals, and the temperature coefficient of the surface tension for fifteen metals. It is worth noting that some of the metals chosen by the author to check the performance of his model are far "simple". As an outstanding closure to his review, the author briefly comments on a universal melting diagram, showing three non-equilibrium states: a crystal supersaturated with vacancies, the undercooled liquid, and the amorphous solid. The diagram (normalized temperature $T / T_{\mathrm{m}}$ versus vacancy concentration $C_{\mathrm{v}}$ ) shows a solid-liquid critical point $\left(T^{*} / T_{\mathrm{m}}, C_{\mathrm{v}}{ }^{*}\right)$ below which only the amorphous solid can exist. Checking the validity and usefulness of this melting diagram either experimentally or by means of the power ab initio theoretical tools now available, will be worthwhile.

Materials processing techniques that make use of liquid metals, such as infiltration or brazing, require a deep knowledge on the basic properties of the metal and of the interface generated between the metal and other present phases, generally solid and of a ceramic or metallic nature. In the review by Professor Eustathopoulos [2], several examples have been given showing how simple wetting experiments can provide very useful information on the basic mechanisms involved in materials processing by infiltration or in joining similar or dissimilar materials by brazing alloys. In this paper, the author offers a critical overview on the kinetics of the spreading of liquid metals on solid surfaces, considering both possible cases: non-reactive wetting and reactive wetting. Current existing models aiming to explain the kinetics of wetting in reactive systems are discussed based on the most recent findings. The author points out that a general description of reactive wetting, taking into account both the localized and delocalized reaction, is still lacking. As for the experimental methods used in wettability studies, great improvements are expected to be made in the near future, including the development of new devices where sessile drop experiments can be coupled in the same chamber with high temperature surface analysis techniques.

Understanding liquid metals is not only important for the manufacturing industry but also for other fields such as geophysics or astrophysics. Indeed, a deep knowledge of the liquid structure and properties at very high pressures is crucial for the development of physically based models of planetary cores. And such models can be very complex since they can involve, for example, the generation of magnetic fields due to the convection flows of liquid metals. In particular, the study of turbulent convection in liquid metals is getting increasing relevance in the field of Geophysics. This is the topic of the article by Riveiro et al. [3]. In this article the authors present experiments at a 
laboratory scale to benchmark complex hydrodynamics codes with magnetic fields. Experiments presented here are done in liquid gallium. Such experiments together with numerical models provide parameters that can be used in larger scale simulation tools.

Despite the large number of studies on liquids, new phenomena still arise that remain almost unexplored. $\mathrm{Zu}$ et al. [4] propose the possibility of a liquid-liquid transition for certain materials. Phase diagrams of ordinary alloys do not show any line above liquidus. However, here, the author shows evidence of a transition induced by temperature in several metals within the liquid state. This transition occurs at ordinary pressures, but at temperatures well-above the melting temperature. Novel experiments are devised to show this transition, such as internal friction, firstly applied for liquids by the author. Moreover, those solids resulting from the liquid after the transition have characteristics that can differ from those obtained from solidification of the liquid before the transition.

Recent experiments have detected the presence of icosahedral superclusters in undercooled liquids. These superclusters survive above melting temperature $\boldsymbol{T}_{\mathrm{m}}$ as their surface atoms have the same fusion heat as their core atoms, and are melted by liquid homogeneous and heterogeneous nucleation in their core, depending on superheating time and temperature. They act as heterogeneous growth nuclei of the crystallized phase at a temperature $\boldsymbol{T}_{\mathrm{c}}<T_{\mathrm{m}}$, contributing to the reduction of the critical barrier that becomes smaller than that of crystals with the same number of atoms $\boldsymbol{n}$. As Tournier [5] discusses in his review, after weak superheating, the most stable superclusters with $\boldsymbol{n}=13,55,147,309$, and 561 survive or melt and determine $\boldsymbol{T}_{\mathrm{c}}$ during undercooling, depending on $\boldsymbol{n}$ and sample volume. The experimental nucleation temperatures $\boldsymbol{T}_{\mathrm{c}}$ of 32 liquid elements and the supercluster melting temperatures are predicted with sample volumes varying by 18 orders of magnitude. In his analysis, Tournier utilizes the classical Gibbs free energy change, adding an enthalpy saving related to the Laplace pressure change derived from supercluster formation, which he quantifies for $\boldsymbol{n}=13$ and 55, and shows to be proportional to the inverse of the supercluster radius for $\boldsymbol{n}>55$. The transformation of superclusters in crystals occurs for a radius between the critical radius for crystal growth and that for supercluster growth as the superclusters have a much lower density than crystals. The Gibbs free energy change from the liquid state to crystal becomes smaller than that of the superclusters just above its maximum at the crystal critical radius.

A variety of families of liquid metallic alloys are being developed that, when solidified, form amorphous materials that are expected to give rise to revolutionary technologies [11]. Wang et al. [6] discuss, in their paper, the glass-forming ability in the ternary $\mathrm{Al}-\mathrm{Cu}-\mathrm{Y}$ system. In particular, they identify the regions of the phase diagram where glasses are formed by means of thermodynamic calculations based on Miedema's model and Alonso's method. They succeed in locating a hexagonal region within the ternary phase diagram where metallic glass formation is favored energetically. In addition, they concluded $\mathrm{Al}_{72} \mathrm{Cu}_{10} \mathrm{Y}_{18}$ to be the optimal composition for glass formation. Available experiments seem to support their predictions. They also claim that the semi-empirical methods they have used might be greatly useful to find regions of phase diagrams of multicomponent alloy systems where glass formation is most probable.

$\mathrm{Al}-\mathrm{Si}$ and Al-Mg-Si alloys are two of the most important families of aluminum alloys, both offering a wide variety of technological applications. In particular, Al-Si alloys are most popular in automotive applications. Additionally, $\mathrm{Mg}$ is added to improve mechanical properties through formation of finely dispersed coherent $\mathrm{Mg}_{2} \mathrm{Si}$ precipitates. When the $\mathrm{Si}$ content is high enough, and depending on 
processing conditions, large primary Si crystals and/or acicular Al-Si eutectic can me formed. Both are highly detrimental as far as final properties of the alloy are concerned. In order to modify this microstructure, several elements and/or alloys are added to the melt that during the solidification process modify the acicular eutectic to a less detrimental globular eutectic acting also as a high-efficiency heterogeneous nucleation substrate for the primary $\mathrm{Si}$ so reducing their size. Zhu et al. [7] consider the performance of AlP alloy as a modifier addressing the important technological problem of whether the order in which $\mathrm{Mg}$ and $\mathrm{AlP}$ are added to the melt matters. Extensive experimental studies and numerical calculations by means of $a b$ initio molecular dynamics simulations allow the authors to conclude: (a) when pure $\mathrm{Mg}$ is added to the Al-Si melt before the Al-P alloy, Mg-Si clusters form that coexist with P-Al clusters. During solidification, the P-Al clusters precipitate as AlP leading to the formation of small primary Si particles; (b) instead, when the AlP alloy is added first, $\mathrm{P}-\mathrm{Al}$ clusters evolve into $\mathrm{P}-\mathrm{Mg}$ clusters on $\mathrm{Mg}$ addition. In the solidification process, $\mathrm{P}$ precipitates as $\mathrm{Mg}_{3} \mathrm{P}_{2}$, rather than AlP. Thus, nucleation of primary $\mathrm{Si}$ particles is diminished due to the absence of AlP.

Perhaps the reason for the lack of understanding of some aspects of liquids is that, experimentally, the study of liquids poses some significant challenges, particularly when considering high pressures. De Ressegier and co-authors show [8] how lasers, together with different characterization techniques, such as optical shadowgraphs and scanning electron micrographs, can provide information about liquid transitions induced by high pressures and strain rates. The authors show clear evidence for this transition for the case of aluminum, as well as tin. Iron, however, does not show a complete melt, although there seems to be a layer of melted material below the surface. These results have important consequences for understanding fundamental issues, such as the Earth's core.

Room-temperature liquid-metal droplets are important for many applications: energy harvesting, self-healing composites, soft electrodes, micro-pumps, interconnects, liquid marbles, switches and relays, etc. An eutectic gallium and indium alloy (EGaIn, 75\% gallium and 25\% indium) has been used in some of these applications by enabling that a thin oxide skin forms spontaneously on the metal at ambient conditions, which allows EGaIn to form stable shapes that would otherwise be prohibited by surface tension. The paper [9] demonstrates a molding technique for producing spheres composed of eutectic gallium-indium (EGaIn) with diameters ranging from hundreds of microns to a couple of mm. The technique starts by spreading EGaIn across an elastomeric sheet featuring cylindrical reservoirs defined by replica molding. Subsequent exposure to acid removes the oxide and causes the metal to bead up into a sphere, with a size dictated by the volume of the reservoirs. EGaIn spheres can subsequently be embedded or encased in polymer matrices using this technique. This technique can be used for producing many spheres in parallel, with any desired two-dimensional spatial arrangement.

Liquids containing particles of nm size (nano-fluids) have attracted considerable attention with the goal of obtaining excellent thermal conductors [10]. Nano-fluids are interesting due to the fact that the poor thermal conductivity of a fluid (or liquid) medium can be supplemented by the high thermal conductivity of the suspended nanoparticles. In paper [10], the authors review of the preparation, physicochemical properties, and the suppression of chemical reactivity to water and oxygen of LSnanop (Liquid Sodium containing nanoparticles of titanium). The presence of small amounts of Ti nanoparticles ( $2 \%$ as the atomic $\%$ of $\mathrm{Ti}, 6.92 \times 10^{-7}$ as the number fraction of Ti nanoparticles, and 0.0088 as their volume fraction) makes physicochemical properties change considerably, compared to 
liquid Na. The ideas behind LSnanop can be extended to produce many other interesting and valuable materials, such as ferromagnetic liquid sodium, by properly selecting the convenient nanoparticles to be dispersed in the liquid metal.

Despite the rich variety of issues discussed in the ten papers published in this Special Issue, there is still a wealth of issues that deserve at least a brief comment in this Introduction. The study of liquid metals under microgravity conditions is throwing light on several key aspects of solidification $[12,13]$. The use of liquid-metal solutions to produce greener crystalline silicon is expected to provide a practical way to produce this old but still-essential material [14]. An issue not yet fully understood is the positive temperature coefficient of surface tension that some liquid metals (more specifically, alloys) show [15,16]. Significant progress in a classical issue of enormous relevance, i.e., the structure of liquid metals, is being attained due to the combined use of synchrotron X-ray diffraction and molecular dynamics simulations. For instance, it has been found that in some liquid metals, the interatomic distance contracts on heating, while at the same time coordination decreases [17]. All this modestly illustrates the strong breath of research that still exists in the field of liquid metals.

\section{Acknowledgments}

This work was supported by the European Union Seventh Framework Programme (FP7/2007-2013) under grant agreement 280464, project "High-frequency Electro-Magnetic technologies for advanced processing of ceramic matrix composites and graphite expansion" (HELM) and "Ministerio de Economía y Competitividad" through project MAT2011-25029.

\section{Conflicts of Interest}

The authors declare no conflict of interest.

\section{References}

1. Wang, L. Estimating the Energy State of Liquids. Metals 2014, 4, 570-585.

2. Eustathopoulos, N. Wetting by Liquid Metals-Application in Materials Processing: The Contribution of the Grenoble Group. Metals 2015, 5, 350-370.

3. Ribeiro, A.; Fabre, G.; Guermond, J.-L.; Aurnou, J.M. Canonical Models of Geophysical and Astrophysical Flows: Turbulent Convection Experiments in Liquid Metals. Metals 2015, 5, 289-335.

4. Zu, F.Q. Temperature-Induced Liquid-Liquid Transition in Metallic Melts: A Brief Review on the New Physical Phenomenon. Metals 2015, 5, 395-417.

5. Tournier, R.F. Crystallization of Supercooled Liquid Elements Induced by Superclusters Containing Magic Atom Numbers. Metals 2014, 4, 359-387.

6. Wang, Q.; Li, J.; Liu, J.; Liu, B. Predicting Composition Dependence of Glass Forming Ability in Ternary Al-Cu-Y System by Thermodynamic Calculation. Metals 2014, 4, 519-529.

7. Zhu, X.; Jiang, W.; Li, M.; Qiao, H.; Wu, Y.; Qin, J.; Liu, X. The Effect of Mg Adding Order on the Liquid Structure and Solidified Microstructure of the Al-Si-Mg-P Alloy: An Experiment and ab Initio Study. Metals 2015, 5, 40-51. 
8. De Rességuier, T.; Loison, D.; Dragon, A.; Lescoute, E. Laser Driven Compression to Investigate Shock-Induced Melting of Metals. Metals 2014, 4, 490-502.

9. Mohammed, M.G.; Xenakis, A.; Dickey, M.D. Production of Liquid Metal Spheres by Molding. Metals 2014, 4, 465-476.

10. Itami, T.; Saito, J.-i.; Ara, K. The Promising Features of New Nano Liquid Metals-Liquid Sodium Containing Titanium Nanoparticles (LSnanop). Metals 2015, 5, 1212-1240.

11. Demetriou, M.D.; Launey, M.E.; Garrett, G.; Schramm, J.P.; Hofmann, D.C.; Johnson W.L.; Ritchie, R.O. A damage-tolerant glass. Nat. Mater. 2011, 10, 123-128.

12. Gu, J.; Fahrenkrug E.; Maldonado, S. Direct Electrodeposition of Crystalline Silicon at Low Temperatures. J. Am. Chem. Soc. 2013, 135, 1684-1687.

13. Egry, I.; Lohoefer, G.; Jacobs, G. Surface Tension of Liquid Metals: Results from Measurements on Ground and in Space. Phys. Rev. Lett. 1995, 75, 4043.

14. Ratke, L.; Diefenbach, S. Liquid immiscible alloys. Mater. Sci. Eng. R Rep. 1995, 15, 263-347.

15. Lee, J.; Shimoda, W.; Tanaka, T. Temperature dependence of surface tension of liquid Sn-Ag, In-Ag and In-Cu alloys. Measurement Sci. Technol. 2005, 2, 438-442.

16. Lee, J.; Shimoda, W.; Tanaka, T. Surface Tension and its Temperature Coefficient of Liquid Sn-X (X = Ag, Cu) Alloys. Mater. Trans. 2004, 45, 2864-2870.

17. Lou, H.; Wang, X.; Cao, Q.; Zhang, D.; Zhang, J.; Hu, T.; Mao, H.K.; Jiang, J.Z. Negative expansions of interatomic distances in metallic melts. PNAS 2013, 102, 1068-1072.

(C) 2015 by the authors; licensee MDPI, Basel, Switzerland. This article is an open access article distributed under the terms and conditions of the Creative Commons Attribution license (http://creativecommons.org/licenses/by/4.0/). 